\title{
Connection between two forms of extra dimensional metrics revisited
}

\author{
Mikhail Z. Iofa ${ }^{1}$ \\ Skobeltsyn Institute of Nuclear Physics \\ Moscow State University \\ Moscow 119992, Russia
}

\begin{abstract}
5D cosmological model with 3-brane with matter is considered. The brane divides bulk in two AdS half spaces. Geometry of the model can be described by two types of coordinates: in the first setting the metric is static and the brane is moving in the bulk, in the second approach the metric is time-dependent and the brane is located at a fixed position in the bulk. Coordinate transformation connecting two coordinate systems is constructed.
\end{abstract}

Recently cosmological models with extra dimensions have attracted a lot of attention [1]). We consider a 5D model with infinite extra dimension with the brane with matter embedded in the bulk. The brane divides the bulk in two AdS spaces at both sides of the brane. The model can be considered in two alternative approaches. In the first approach the bulk is static, and the brane is moving in the bulk $[2,3,4,5,6]$. In the second approach metric of the model is time-dependent, and the brane is located at a fixed position in extra dimension $[7,8]$. Coordinate transformation between two settings is not trivial and was discussed previously [6, 10] and recently [11]. Below, omitting various generalizations of the model, we construct transformation between two pictures in a simple and straightforward way.

The energy-momentum tensor on the brane is

$$
\tau_{\mu}^{\nu}=\operatorname{diag}\{-\hat{\rho}-\hat{\sigma}, \hat{p}-\hat{\sigma}, \hat{p}-\hat{\sigma}, \hat{p}-\hat{\sigma}\},
$$

where $\hat{\rho}$ and $\hat{p}$ are the energy and momentum densities of matter on the brane, $\hat{\sigma}$ is the tension of the brane.

It is convenient to define the normalized energy density and tension of the brane

$$
\rho=\frac{\kappa^{2} \hat{\rho}}{6}, \quad \sigma=\frac{\kappa^{2} \hat{\sigma}}{6}
$$

where $\hat{\rho}$ and $\hat{\sigma}$ have dimension $[\text { mass }]^{4}$ and $\rho$ and $\sigma$ have dimension $[\text { mass }]^{1}$. Parameter $\mu$ having dimension [mass] is defined via the $5 \mathrm{D}$ cosmological constant $\Lambda$ as $\mu^{2}=\kappa^{2}|\Lambda| / 6$, where $\kappa^{2}=8 \pi / M^{3}$ is the $5 \mathrm{D}$ gravitational coupling constant, and $M$ is the $5 \mathrm{D}$ gravitational scale.

In approach with the static metric, the metric is

$$
d s^{2}=-f(R) d T^{2}+\frac{d R^{2}}{f(R)}+\mu^{2} R^{2} d x^{a} d x_{a} \equiv g_{M N} d x^{M} d x^{N},
$$

where

$$
f(R)=\mu^{2} R^{2}-\frac{P}{R^{2}}
$$

\footnotetext{
1e-mail: iofa@theory.sinp.msu.ru
} 
$R$ and $T$ have dimension $[\text { mass }]^{-1}$.

In a parametric form trajectory of the brane is defined through the proper time $t$ on the brane as $R=R_{b}(t), T=T_{b}(t)$, where $-f\left(R_{b}\right) \dot{T}_{b}^{2}+f^{-1}\left(R_{b}\right) \dot{R}_{b}^{2}=-1$. It follows that

$$
\dot{T}=\xi \frac{\sqrt{f\left(R_{b}\right)+\dot{R}_{b}^{2}}}{f\left(R_{b}\right)},
$$

where $\xi= \pm 1$. The metric on the brane is $d s^{2}=-d t^{2}+\mu^{2} R_{b}^{2}(t) d x^{a} d x_{a}$.

Trajectory of the brane with fixed 3D coordinates is determined by solving the junction conditions for the metric on the brane. The junction conditions on the brane are $[4,3,7,8,9]$

$$
\left[h_{i}^{k} \nabla_{k} n_{j}\right]=\tau_{i j}-\frac{1}{3} \tau h_{i j} .
$$

Here $h_{i j}=g_{i j}-n_{i} n_{j}$ is the induced metric on the brane, $v^{i}= \pm\left(\dot{T}_{b}, \dot{R}_{b}, 0\right)$ and $n_{i}= \pm\left(-\dot{R}_{b}, \dot{T}_{b}, 0\right)$ are velocity and normal vector to the brane $\tau_{i j}=(\rho+p) v_{i} v_{j}+p h_{i j},[\mathrm{X}]$ denotes the difference of expressions calculated at the opposite sides of the brane.

From the spatial components $(a, b=1,2,3)$ of the junction conditions follows the generalized Friedmann equation $[2,3,4,7,8,9,1]$

$$
\left(\frac{\dot{R}_{b}}{R_{b}}\right)^{2}=-\mu^{2}+(\rho+\sigma)^{2}+\frac{P}{R_{b}^{4}} .
$$

In the second approach we consider a class of non-static metrics $[7,8]$

$$
d s^{2}=-n^{2}(y, t) d t^{2}+a^{2}(y, t) d x^{a} d x_{a}+d y^{2} .
$$

The brane is located at a fixed position in the extra coordinate, which we set $y=0$. The function $n(y, t)$ is normalized by condition $n(0, t)=1$. For simplicity we consider a spatially flat brane. Here

$$
\begin{aligned}
& a^{2}(y, t)=\frac{a^{2}(0, t)}{4}\left[e^{2 \mu|y|}\left(\left(\frac{\rho+\sigma}{\mu}-1\right)^{2}+\frac{\rho_{w}}{\mu}\right)+e^{-2 \mu|y|}\left(\left(\frac{\rho+\sigma}{\mu}+1\right)^{2}+\frac{\rho_{w}}{\mu}\right)\right. \\
& \left.-2\left(\left(\frac{\rho+\sigma}{\mu}\right)^{2}-1+\frac{\rho_{w}}{\mu}\right)\right],
\end{aligned}
$$

and $n(y, t)=\dot{a}(y, t) / \dot{a}(0, t)$. Restriction of the metric (5) to the brane is $d s^{2}=-d t^{2}+a^{2}(t) d x^{a} d x_{a}$. The function $a(t)=a(0, t)$ satisfies generalized Friedmann equation $[7,8]$

$$
H^{2}(t)=-\mu^{2}+(\rho+\sigma)^{2}+\mu \rho_{w}(t)
$$

where

$$
H(t)=\frac{\dot{a}(0, t)}{a(0, t)}, \quad \rho_{w}(t)=\frac{\rho_{w 0}}{a^{4}(0, t)} .
$$

are the Hubble function and the Weyl radiation term. In derivation of (6) $\rho_{w 0}$ appears as an integration constant [8]. Eqs (4) and (7) have the same form. $a^{2}(0, t)$ can be identified with $\mu^{2} R_{b}^{2}(t)$, and the term $\rho_{w}(t)=\rho_{w 0} / a^{4}(t)$ can be identified with the term $P / \mu R_{b}^{4}(t)$. 
In the picture with the static metric we consider the geodesic equations starting at the brane world sheet

$$
\begin{aligned}
& \frac{d^{2} T}{d y^{2}}+2 \Gamma_{T R}^{T} \frac{d T}{d y} \frac{d R}{d y}=0 \\
& \frac{d^{2} x^{a}}{d y^{2}}+2 \Gamma_{b R}^{a} \frac{d x^{b}}{d y} \frac{d R}{d y}=0 \\
& \frac{d^{2} R}{d y^{2}}+\Gamma_{R R}^{R}\left(\frac{d R}{d y}\right)^{2}+\Gamma_{T T}^{R}\left(\frac{d T}{d y}\right)^{2}+\Gamma_{a b}^{R} \frac{d x^{a}}{d y} \frac{d x^{b}}{d y}=0
\end{aligned}
$$

where $y$ is the affine parameter and the Christoffel symbols are

$$
\Gamma_{T R}^{T}=\frac{f^{\prime}}{2 f}, \quad \Gamma_{R R}^{R}=-\frac{f^{\prime}}{2 f}, \quad \Gamma_{T T}^{R}=\frac{1}{2} f f^{\prime}, \quad \Gamma_{a b}^{R}=-\eta_{a b} f \mu^{2} R, \quad \Gamma_{R b}^{a}=\frac{\delta_{b}^{a}}{R} .
$$

Here $(T, R) \equiv\left(T^{ \pm}, R^{ \pm}\right)$are coordinates in the AdS spaces at the opposite sides of the brane. Integrating the geodesic equations, one obtains

$$
\frac{d T^{ \pm}}{d y}=\frac{E^{ \pm}}{f(R)}, \quad \frac{d x^{a}}{d y}=\frac{C^{a}}{\mu^{2} R^{2}}, \quad\left(\frac{d R^{ \pm}}{d y}\right)^{2}=f(R)\left(C^{R \pm}\right)^{2}+E^{ \pm 2}-\frac{C^{a 2} f}{\mu^{2} R^{2}},
$$

where $\left(E^{ \pm}, C^{a}, C^{R \pm}\right)$ are integration parameters. $\left(d T / d y, d R / d y, d x^{a} / d y\right)$ are the components of the tangent vectors to geodesics which we normalize to unity. Imposing the normalization condition

$$
\frac{d x^{M}}{d y} \frac{d x^{M}}{d y} g_{M N}=1, \quad M, N=T, R, a
$$

we obtain that $\left(C^{R \pm}\right)^{2}=1$. We consider solutions of the geodesic equations even in $y$ : $T^{+}(y)=$ $T^{-}(-y), R^{+}(y)=R^{-}(-y)$.

The hypersurface $\left(T, R, x^{a}=0\right)$ is foliated by geodesics that intersect the trajectory of the brane $\left(T_{b}(t), R_{b}(t)\right)$ and at the intersection point are orthogonal to the world sheet of the brane (cf [10]). Parameter $t$ is constant along the geodesics orthogonal to the brane world sheet and can be considered as a parameter labeling the geodesics. Setting $C^{a}=0$, have

$$
\frac{\partial T^{ \pm}(y, t)}{\partial y}=\frac{E \epsilon(y)}{f(R)}, \quad \frac{\partial R^{ \pm}(y, t)}{\partial y}=\alpha \epsilon(y)\left(f(R)+E^{2}\right)^{1 / 2}
$$

where $\alpha= \pm 1$, and $E^{+}=E^{-}=E$.

The normalized velocity vector to the trajectory of the brane and the normal vectors to the hyperplane containing trajectory of the brane $\left(T_{b}(t), R_{b}(t)\right)$ are

$$
\left.v_{b}^{i}=\left(\dot{T}_{b}, \dot{R}_{b}\right)=\left(\xi \sqrt{f\left(R_{b}\right)+\dot{R}_{b}^{2}} / f\left(R_{b}\right), \dot{R}_{b}\right)\right) \quad n_{b}^{i \pm}=\eta \epsilon(y)\left(\frac{\dot{R}_{b}}{f\left(R_{b}\right)}, \xi \sqrt{f\left(R_{b}\right)+\dot{R}_{b}^{2}}\right),
$$

where $\eta= \pm 1$. From (13) we obtain the tangent vector to a geodesic

$$
u^{i \pm}=\left(\frac{E \epsilon(y)}{f(R)}, \alpha \epsilon(y) \sqrt{f(R)+E^{2}}\right)
$$


By construction, the tangent vector to a geodesic at the intersection point with trajectory of the brane is (anti)parallel to the normal to the trajectory of the brane,

$$
\left.u^{i}\right|_{y=0} \| n_{b}^{i}
$$

From this condition it follows that

$$
E=\eta \dot{R}_{b}, \quad \alpha=\xi \eta
$$

Denoting $Q^{i}=(R, T)$ and $u^{i}=\partial Q^{i} / \partial y$, and introducing $a_{j}=u^{i} g_{i j}$, normalization condition (12) with $C^{a}=0$ can be written as

$$
\left.a_{j} \frac{\partial Q^{j}(y, t)}{\partial y}\right|_{t}=1
$$

From this equation we obtain

$$
a_{j}=\frac{\partial y}{\partial Q^{j}}+\varphi(y, t) \frac{\partial t}{\partial Q^{j}}
$$

where $\varphi(y, t)$ is an arbitrary function ${ }^{2}$. Although in a general case I cannot prove that $\varphi=0$, in a special case discussed below it is possible to have $\varphi=0$.

If $\varphi=0,(18)$ yields $\partial y / \partial Q^{i}=g_{i j} \partial Q^{j} / \partial y$, or

$$
\frac{\partial y}{\partial R}=\frac{1}{f(R)} \frac{\partial R(y, t)}{\partial y}, \quad \frac{\partial y}{\partial T}=-f(R) \frac{\partial T(y, t)}{\partial y}
$$

From the equality

$$
d y=\frac{\partial y}{\partial R}\left(\frac{\partial R}{\partial y} d y+\frac{\partial R}{\partial t} d t\right)+\frac{\partial y}{\partial T}\left(\frac{\partial T}{\partial y} d y+\frac{\partial T}{\partial t} d t\right)
$$

it follows that

$$
\frac{\partial y}{\partial R} \frac{\partial R}{\partial t}+\frac{\partial y}{\partial T} \frac{\partial T}{\partial t}=0
$$

Using (19), this relation is written in a form

$$
\frac{\partial T(y, t) / \partial t}{\partial R(y, t) / \partial t}=\frac{1}{f^{2}(R)} \frac{\partial R(y, t) / \partial y}{\partial T(y, t) / \partial y}
$$

Now it is possible to transform the metric (1) to the form (5). The metric (1) is written as $d s^{2}=d y^{2}\left(-f(R) T^{\prime 2}+\frac{R^{\prime 2}}{f(R)}\right)+2 d y d t\left(-f(R) T^{\prime} \dot{T}+\frac{R^{\prime} \dot{R}}{f(R)}\right)+d t^{2}\left(-f(R) \dot{T}^{2}+\frac{\dot{R}^{2}}{f(R)}\right)+\mu^{2} R^{2} d x^{a} d x_{a}$

Using (13) we find that the coefficient at $d y^{2}$ is 1 , using $(13),(19),(21)$ that the coefficient at $d y d t$ is zero, and at $d y^{2}$ is $\dot{R}^{2} / \dot{R}_{b}^{2}=n^{2}(y, t)$.

Let us consider a special case $P=0$ in (1). Integrating the second equation (13), we obtain

$$
R^{ \pm}(y, t)=R_{b}(t) \cosh \mu y+\alpha \sqrt{\frac{\dot{R}_{b}^{2}}{\mu^{2}}+R_{b}^{2}} \sinh \mu|y| .
$$

Using the Friedmann equation with $P=0$

$$
H^{2}=\dot{R}_{b}^{2} / R_{b}^{2}=\rho^{2}+2 \mu \rho
$$

\footnotetext{
${ }^{2}$ This remark is due to I. Tyutin and B. Voronov.
} 
and introducing $\beta=\operatorname{sign}\left(R_{b}(t)\right)$, we rewrite $(23)$ as

$$
R^{ \pm}(y, t)=R_{b}(t)\left(\cosh \mu y+\alpha \beta\left(1+\frac{\rho}{\mu}\right) \sinh \mu|y|\right) .
$$

For correspondence with (6) (with $P=0$ )

$$
a^{2}(y, t)=\frac{a^{2}(0, t)}{4}\left[e^{2 \mu|y|}\left(\frac{\rho}{\mu}\right)^{2}+e^{-2 \mu|y|}\left(\frac{\rho}{\mu}+2\right)^{2}-2 \frac{\rho}{\mu}\left(\frac{\rho}{\mu}+2\right)\right]
$$

we set

$$
\alpha \beta=-1
$$

and (omitting $( \pm)$ ) obtain

$$
R(y, t)=\frac{R_{b}(t)}{2}\left[e^{-\mu|y|}\left(\frac{\rho}{\mu}+2\right)-e^{\mu|y|} \frac{\rho}{\mu}\right] .
$$

Also we have

$$
\frac{d R(y, t)}{d y}=-\epsilon(y) \frac{\mu R_{b}}{2}\left[e^{-\mu|y|}\left(\frac{\rho}{\mu}+2\right)+e^{\mu|y|} \frac{\rho}{\mu}\right] .
$$

Introducing $y_{0}$, such that

$$
e^{\mu y_{0}}=\left(\frac{\rho}{\rho+2 \mu}\right)^{1 / 2}
$$

we express $R(y, t)$ and $R^{\prime}(y, t)$ as

$$
R(y, t)=-\frac{\dot{R}_{b}(t)}{\mu} \sinh \left(\mu|y|+\mu y_{0}\right), \quad R^{\prime}(y, t)=-\epsilon(y) \dot{R}_{b}(t) \cosh \left(\mu|y|+\mu y_{0}\right)
$$

Integrating the first Eq. (13) for $T(y, t)$ with $R(y, t)(26)$, we obtain

$$
T^{ \pm}(y, t)=-\frac{1}{\mu E} \frac{\cosh \left(\mu|y|+\mu y_{0}\right)}{\sinh \left(\mu|y|+\mu y_{0}\right)}+C^{ \pm}(t) .
$$

For $C^{+}(t)=C^{-}(t)=C(t)$ the limits $y=0$ from both sides of the trajectory of the brane are the same.

From the fact that in (22) the coefficient at $d y d t$ is zero it follows that

$$
\dot{T}=\frac{\dot{R} R^{\prime}}{\mu^{4} R^{4} T^{\prime}}=\frac{\dot{R} R^{\prime}}{\mu^{2} R^{2}(\epsilon(y) E)} .
$$

Writing (30) as

$$
T(y, t)=-\frac{R^{\prime}(y, t) / \epsilon(y)}{\mu^{2} R(y, t) E}+C(t)
$$

and taking the time derivative, we obtain

$$
\dot{T}=\frac{\dot{R} R^{\prime}}{\mu^{2} R^{2}(\epsilon(y) E)}-\frac{1}{\mu^{2} R} \frac{d}{d t}\left(\frac{R^{\prime} \epsilon(y)}{E}\right)+\dot{C}
$$


The first term in the rhs of (33) written as

$$
\frac{\dot{R} R^{\prime}}{\mu^{2} R^{2}(\epsilon(y) E)}=\frac{\dot{R} R^{\prime}}{\left(\mu^{2} R^{2}\right)^{2} T^{\prime}}
$$

is the same as in (21), which was obtained assuming that in (18) $\varphi=0$. Remarkably, substituting explicit expressions (29) for $R$ and $R^{\prime}$, we find that the second term on the rhs of (33) is independent of $y$

$$
\frac{1}{\mu^{2} R} \frac{d}{d t}\left(\frac{R^{\prime} \epsilon(y)}{E}\right)=\frac{\eta \dot{y}_{0}(t)}{\dot{R}_{b}(t)} .
$$

Choosing

$$
\dot{C}=\eta \frac{\dot{y}_{0}}{\dot{R}_{b}}
$$

we obtain $\dot{T}$ as in (31). In the limit $y=0$ we have

$$
\left.\dot{T}(y, t)\right|_{y \rightarrow 0}=-\eta \frac{\rho+\mu}{\mu^{2} R_{b}}=\dot{T}_{b}(t) .
$$

In the radiation-dominated period, conservation equation for the energy density on the brane is

$$
\dot{\rho}=-4 \rho \dot{R}_{b} / R_{b}
$$

Using this equation, we have

$$
\dot{y}_{0}=-\frac{4 \rho}{H}, \quad \dot{T}_{b}=-\eta \frac{\rho+\mu}{\mu^{2} R_{b}} .
$$

From (26), using the conservation equation for $\rho$, we obtain

$$
\dot{R}(y, t)=\frac{\dot{R}_{b}}{2}\left[e^{\mu|y|} \frac{3 \rho}{\mu}-e^{-\mu|y|}\left(\frac{3 \rho}{\mu}-2\right)\right] .
$$

Jacobian of transformation from $T, R$ to $t, y$ is

$$
J=\dot{T} R^{\prime}-\dot{R} T^{\prime}=\epsilon \eta \frac{\dot{R}}{\dot{R}_{b}} .
$$

Although both $\dot{R}$ and $\dot{R}_{b}$ are non-zero, the expressions for $\dot{T}$ and $T^{\prime}$ contain $R(y, t)$ in denominator. $R(y, t)$ is zero for $e^{2 \mu|y|}=(\rho+2 \mu) / \rho$, and transformation from $(T, R)$ to $(t, y)$ is valid for $e^{2 \mu|y|}<$ $1+2 \mu / \rho$. In the region $e^{2 \mu|y|}>1+2 \mu / \rho R(y, t)$ can be defined as

$$
R(y, t)=\frac{R_{b}(t)}{2}\left[e^{\mu|y|} \frac{\rho}{\mu}-e^{-\mu|y|}\left(\frac{\rho}{\mu}+2\right)\right],
$$

which is a positive and increasing function $y$.

I would like to thank I. Tyutin and B. Voronov for helpful discussion. 


\section{References}

[1] R. Maartens, Living Rev. Rel.7, 7 (2004), [arXiv:gr-qc/0312059], Brane-world gravity.

[2] P.Kraus, JHEP 9912, (1999) 011 [arXiv:hep-th/9910149], Dynamics of Anti-de Sitter Domain Walls.

[3] H.Collins and B.Holdom, Phys. Rev.D62, 105009, (2000), [arXiv:hep-ph/0003173], Brane Cosmologies without Orbifolds.

[4] H.A. Chamblin and H.S. Reall, Nucl. Phys. B562, 133 (1999), [arXiv:hep-th/990322], Dynamic Dilatonic Domain Walls.

[5] D.Birmingham, Class. Quant. Grav. 16 , 1197 (1999), [arXiv:hep-th/9808032], Topological Black Holes in Anti-de Sitter Space.

[6] P. Bowcock, C. Charmousis, and R. Gregory, Class. Quant. Grav. 17, 4745 (2000), [arXiv:hepth/0007177], General brane cosmologies and their global spacetime structure.

[7] P. Binetruy, C. Deffayet and D. Langlois, Nucl. Phys. B565, 269 (2000), [arXiv:hep-th/9905012], Non-conventional cosmology from a brane-universe .

[8] P. Binetruy, C. Deffayet, U. Ellwanger and D. Langlois, Phys. Lett. B477, 285, (2000), [arXiv:hep-th/9910219]. Brane cosmological evolution in a bulk with cosmological constant.

[9] T. Shiromizu, K. i. Maeda and M. Sasaki, Phys. Rev. D62, 024012 (2000), [arXiv:gr-qc/9910076], The Einstein equations on the 3-brane world.

[10] S. Mukohyama, T. Shiromizu, K-i. Maeda, Phys. Rev. D62 (2000) 024028, [arXiv:hepth/9912287] Global structure of exact cosmological solutions in the brane world.

[11] W.-H. Wu and C.-H. Yang, [arXiv:1204.1546], Local and global structure of domain wall spacetimes. 